Ann. Biol. anim. Bioch. Biophys., I973, 13 (4), 6II-626.

\title{
ALCALOSE INDUITE PAR LE NITRATE CHEZ LA POULE ET GARACTÉRISTIQUES DE L'CEUF
}

\author{
B. SAUVEUR \\ avec la collaboration technique de $\mathrm{M}^{\mathrm{me}} \mathrm{H}$. Antorne et M. J. RocARD \\ Station de Recherches avicoles, \\ Domaine de l'Orfrasière, I. N. R. A., \\ B.P. 1 Nouzilly, 37380 Monnaie
}

\section{RÉSUMÉ}

Le but de cet essai est d'étudier si l'ion nitrate, générateur d'alcalose chez les Mammifères, exerce la même action chez la Poule et modifie les caractéristiques de l'œuf. Ce nitrate est incorporé au taux de $\mathrm{I}, 5 \mathrm{p}$. Ioo dans un aliment à teneur en chlore réduite $(\mathrm{Cl}=0, \mathrm{I} 2 \mathrm{p}$. IOO) par rapport à celle du régime témoin $(\mathrm{Cl}=0,24 \mathrm{p}$. I00). La durée de l'essai est de 6 semaines, précédées et suivies de 2 semaines pendant lesquelles les trois lots d'animaux consomment le même aliment témoin.

L'addition de nitrate à l'aliment hypochloré entraîne indubitablement une alcalose métabolique avec hypochlorémie qui cessent dans les deux semaines suivant le retour au régime témoin. Cette alcalose s'accompagne d'une diminution des teneurs plasmatiques en potassium et magnésium.

Au niveau de l'œuf les réponses varient suivant le génotype des animaux : chez des poules White Leghorn, la restriction chlorée à elle seule réduit le poids de l'ouf et la quantité de coquille déposée; à l'opposé, le poids de l'ouf reste inchangé et la coquille est très améliorée si ce même régime est distribué à des animaux issus d'un croisement du type Rhode Island Red $\times$ Wyandotte. En aucun cas l'effet d'un apport de nitrate n'est favorable à ces critères, en opposition ce qu'on aurait pu attendre à la vue des résultats enregistrés eu niveau sanguin.

Ces résultats sont comparés à ceux déjà obtenus chez les mammifères et discutés en fonction des rapports qui existent entre l'équilibre acido-basique et la formation de l'œuf chez les Oiseaux. On recommande de tenir compte du génotype des animaux avant de modifier la teneur en anions des régimes.

On sait que la formation de la coquille de l'œuf dépend de l'équilibre acidobasique de la poule (MoNGIN, I968). Il a été montré en particulier qu'une augmentation chronique de la teneur du plasma sanguin en bicarbonate s'accompagne, dans la quasi-totalité des cas, d'une augmentation du dépôt de carbonate de calcium au 
niveau de la coquille mais également d'une légère altération de la structure physique de 1'albumen (SAUveUR, I970).

Un tel accroissement de la bicarbémie peut être obtenu chez la Poule en réduisant l'ingestion de chlore (MONGIN, SAUVEUR et AmIN, I972). Par ailleurs, on sait que, chez les mammifères, l'administration de nitrate engendre également une alcalose hypochlorémique (HolıIDAy, I955 ; Gulyassy et al., I962 ; WIL,SON et Simmons, I970). C'est pourquoi nous avons étudié les effets d'une supplémentation de l'aliment en nitrate sur l'équilibre acido-basique de la poule et la qualité de l'œuf, indépendamment de toute variation du contenu en cations du régime.

\section{MATÉRIEL, ETT MÉTHODES}

\section{Animaux}

L'expérience a été effectuée à l'aide de 30 poules dont $\mathrm{I}_{5}$ de type White Leghorn (Heisdorf Nelson) et $\mathrm{I}_{5}$ issues d'un triple croisement Rhode Island Red $\times$ Wyandotte (Selaf M5I9). Les animaux, âgés de 8 mois lors de la première phase expérimentale, étaient logés en cages individuelles dans un bâtiment à température et hygrométrie constantes.

\section{TABLEAU I}

Composition des régimes

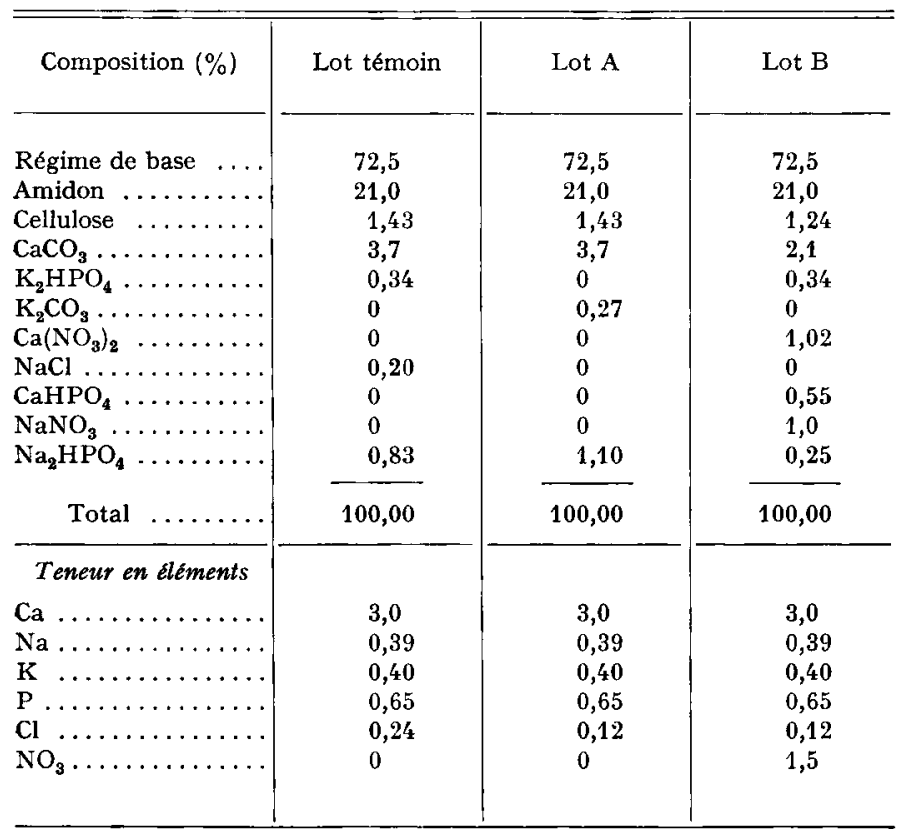

Régime de base : (p. 100)

Amidon 31 ; Gluten maîs 11 ; Huile maïs 1 ; Farine luzerne 8 ; Soja (44) 12 ; Poisson (Pérou) 2 ; Phosph. bicalc. 2 ; $\mathrm{CaCO}_{3} 4,5$; Mélange vitamin. 1. 


\section{Groupes et régimes expérimentaux}

Pendant une première période de deux semaines, toutes les poules ont consommé le même aliment témoin dépourvu de nitrate et contenant $0,24 \mathrm{p}$. Ioo de chlore (tabl. I).

A la fin de cette période, 3 groupes de ro animaux (5 de chaque souche) ont été constitués :

- groupe $\mathrm{T}$ (témoin) : même régime que durant la première période

- groupe A......... régime témoin à teneur en chlore réduite $(0,12$ p. I00)

- groupe $\mathrm{B} . \ldots \ldots \ldots$. régime $\mathrm{A}$ supplémenté en nitrate $\left(1,5 \mathrm{p}\right.$. Ioo de $\mathrm{NO}_{3}$ sous forme de $\mathrm{NaNO}_{3}$ et $\left.\mathrm{de} \mathrm{Ca}\left(\mathrm{NO}_{3}\right)_{2}\right)$

Le détail de composition des mélanges minéraux est donné au tableau $\mathbf{I}$. Ces mélanges ont été calculés de telle sorte que les apports de cations ( $\mathrm{Na}, \mathrm{K}, \mathrm{Ca}$ ) soient égaux et constants dans les 3 régimes.

La distribution de ces trois régimes a été effectuée pendant six semaines consécutives. témoin.

Pendant une dernière période de deux semaines, les 30 poules ont à nouveau reçu le régime

Pour chacune des trois périodes, les poules ont consommé leur aliment ad libitum.

\section{Mesures effectuées}

I. Sang.

Toutes les deux semaines une prise de sang $(4 \mathrm{ml})$ a été effectuée dans la veine alaire de chaque poule dans l'heure suivant une oviposition; cette précaution élimine les variations de l'ionogramme sanguin dues à la formation de l'ouf (Mongin et LACAssagne, I966 ; Hodges, 1970). Le $\mathrm{pH}$ et la $p \mathrm{CO}_{2}$ ont été mesurés suivant la technique décrite par Mongin et LACASSAGNE, (x966); nous avons calculé la concentration des ions bicarbonate au moyen de l'équation d'Hasselbach-Henderson en prenant le pK' égal à 6,9I d'après HELBAckA et al, (I964) et la constante de solubilité du $\mathrm{CO}_{2}$ dans le plasma à $4 \mathrm{I}^{\circ} \mathrm{C}$ égale à $0,0282 \mathrm{mM} / 1 / \mathrm{mmHg}$.

Après centrifugation du sang à l'abri de l'air, la teneur du plasma en $\mathrm{Na}, \mathrm{K}$ et $\mathrm{Ca}$ a été déterminé par spectrophotométrie de flamme (Eppendorf), celle en $\mathrm{Mg}$ par spectrophotométrie d'adsorption atomique (Hilger et Watts). La chlorémie a été appréciée par méthode potentiométrique (Electrode Radiometer $\mathrm{P}_{4} \mathrm{Or}$ ) et la pression osmotique par mesure de l'abaissement du point de congélation (osmomètre Fiske).

\section{Euf.}

Nous avons enregistré sur 3 œufs par poule et par semaine le poids de l'œuf, le poids de coquille par Ioo $\mathrm{cm}^{2}$ de surface ( $($ index de coquille " de MoNGIN, I965) ainsi que la hauteur de l'albumen épais exprimée en unités de HaUgh (1937).

\section{RÉSULTATS}

\section{A. - Au niveau du sang}

L'analyse de variance des résultats enregistrés pour les paramètres sanguins ne fait apparaître aucun effet significatif d'interaction entre la souche de l'animal et lè régime alimentaire distribué. C'est pourquoi les deux souches de poules sont ici considérées en commun. La figure I donne ainsi les variations de l'équilibre acidobasique du sang et de la chlorémie.

La diminution de 50 p. Ioo de la teneur en chlore de l'aliment (lot A) n'entraîne pas d'évolution du $\mathrm{pH}$ sanguin différente de celle observée dans le groupe témoin. Cependant, durant les quatre premières semaines de traitement, la bicarbémie du groupe $A$ est légèrement supérieure à celle du lot témoin $T$ : il semble donc que cette restriction chlorée entraîne une légère alcalose mettabolique transitoire. Elle se tra- 
duit en outre par un abaissement de la chlorémie voisin de 6 méq/1 qui est supprimé dès que les poules reçoivent à nouveau le régime témoin.

L'adjonction à l'aliment contenant $\mathrm{O}, \mathrm{I} 2 \mathrm{p}$. Ioo de $\mathrm{Cl}$ d'une quantité importante de $\mathrm{NO}_{3}$ ( $\mathrm{I}, 5$ p. Ioo - régime $\mathrm{B}$ ) accentue très nettement l'alcalose métabolique. Ceci se traduit par une augmentation $\mathrm{du} \mathrm{pH}$ et de la bicarbémie sans variation importante de la $p \mathrm{CO}_{2}$ sanguine. L'effet le plus net de l'apport de nitrate est un abaissement de la chlorémie voisin de ro méq/1. Cette hypochlorémie cesse lorsque les animaux reçoivent à nouveau le régime témoin; simultanément, la bicarbémie redevient normale.

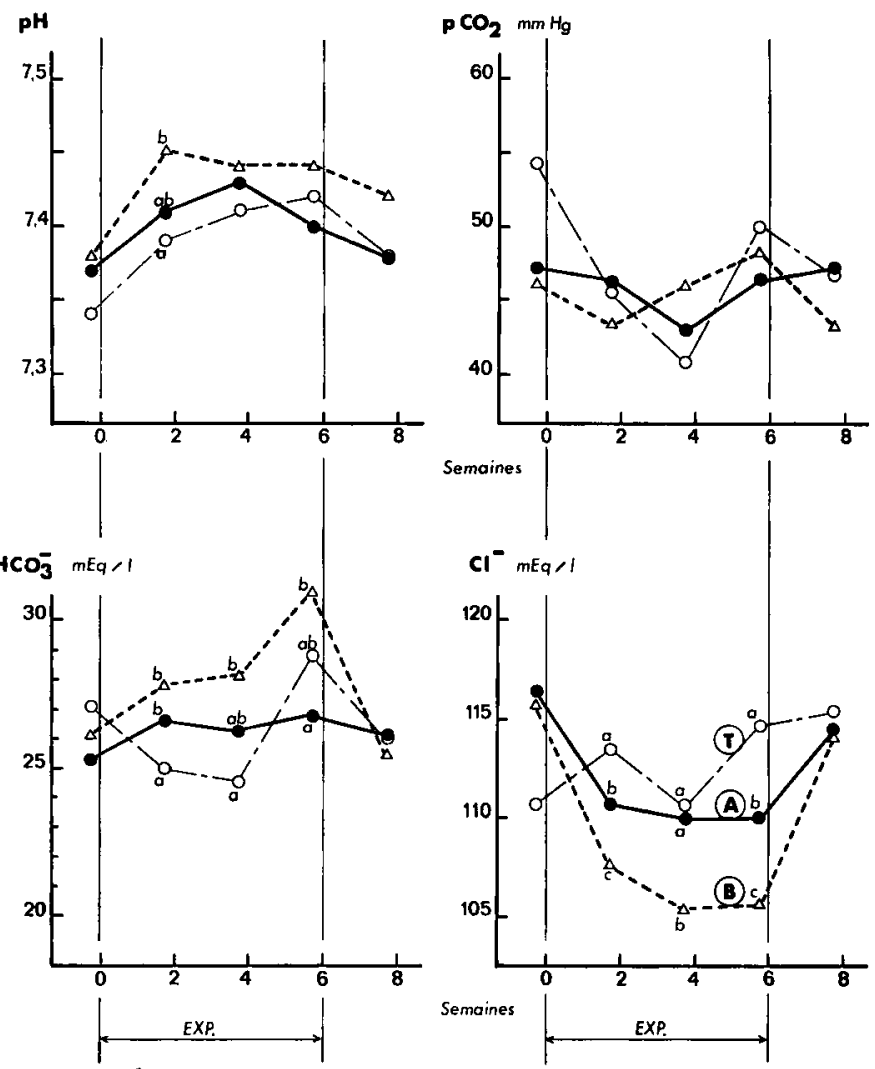

FIG. I. - Équilibre acido-basique du sang et chlorémie chez les animaux recevant des régimes à 0,24 p. 100 de chlore (lot témoin $\mathrm{T}$ ), 0,12 p. 100 de chlore (lot A) ou 0,12 p. 100 de chlore plus 1,5 p. 100 de nitrate (lot $\mathrm{B}$ )

$$
\begin{aligned}
& \circ-\cdots \text { lot témoin } \mathrm{T} \\
& \square \ldots \ldots \text { lot } \mathrm{A} \\
& \mathrm{B}
\end{aligned}
$$

$a, b, c:$ pour chaque paramètre, les valeurs d'une même semaine non suivies des mêmes lettres, sont différentes entre elles $(\mathrm{P}<0,005)$. En l'absence d'indication, les valeurs d'une même semaine ne diffèrent pas de façon significative.

L'effet des trois régimes sur la teneur en cations et la pression osmotique du plasma est reporté au tableau 2. Pour le sodium on constate, durant les deux premières semaines d'expérience, une chute de concentration identique pour les trois lots ; 
par la suite, la natrémie semble être légèrement plus élevée en $A$ (I 48,6 méq/1) et $B$ (I48,2 méq/1) que chez le lot témoin (I46,7 méq/1) mais les différences sont peu importantes. L'action du nitrate alimentaire sur la teneur plasmatique en potassium paraît plus nette : deux semaines après le début de l'expérience, la kaliémie du lot $B$ est inférieure de ro $\mathrm{p}$. Ioo à celle du lot témoin alors qu'elle n'est pas réduite par la seule restriction chlorée (lot $\mathrm{A}$ ). Cette différence se retrouve après 6 semaines d'essai et disparaît après retrait des nitrates de l'aliment.

\section{TABLEAU 2}

Teneur en cations et pression osmotique du plasma sanguin lors de l'alcalose induite par le nitrate chez la Poule (moyenne calculées sur 8 valeurs)

\begin{tabular}{|c|c|c|c|c|c|}
\hline \multirow{3}{*}{ Éléments et Régimes } & \multicolumn{5}{|c|}{ Période } \\
\hline & \multirow{2}{*}{ Préexpérience } & \multicolumn{3}{|c|}{ Expérience (semaines) } & \multirow{2}{*}{ Récupération } \\
\hline & & 2 & 4 & 6 & \\
\hline $\begin{array}{l}\mathrm{Na}^{+}(\mathrm{méq} / \mathrm{l})\left\{\begin{array}{l}\text { Témoin }(\mathrm{T}) \ldots \ldots \\
-\mathrm{Cl}(\mathrm{A}) \ldots \ldots \\
-\mathrm{Cl}+\mathrm{NO}_{3}(\mathrm{~B})\end{array}\right. \\
\text { Valeurs de } \mathrm{F} \ldots \ldots \ldots \ldots \ldots \ldots\end{array}$ & $\begin{array}{l}151,2 \\
153,4 \\
151,1\end{array}$ & $\begin{array}{l}147,9 \\
148,1 \\
148,2 \\
-(1)\end{array}$ & $\begin{array}{r}146,7^{a} \\
148,6^{b} \\
148,2^{b} \\
3,80^{*}\end{array}$ & $\begin{array}{l}147,7 \\
149,7 \\
147,9 \\
1,90\end{array}$ & $\begin{array}{l}148,5 \\
153,4 \\
151,1\end{array}$ \\
\hline $\begin{array}{l}\mathrm{K}^{+}(\text {méq } / \mathrm{l})\left\{\begin{array}{l}\text { Témoin }(\mathrm{T}) \ldots \ldots \\
-\mathrm{Cl}(\mathrm{A}) \ldots \ldots \\
-\mathrm{Cl}+\mathrm{NO}_{3}(\mathrm{~B})\end{array}\right. \\
\text { Valeurs de } \mathrm{F} \ldots \ldots \ldots \ldots \ldots\end{array}$ & $\begin{array}{l}4,47 \\
4,63 \\
4,53\end{array}$ & $\begin{array}{l}4,66^{a} \\
4,68^{a} \\
4,20^{b} \\
5,76^{* *}\end{array}$ & $\begin{array}{l}4,64 \\
4,81 \\
4,73 \\
0,5\end{array}$ & $\begin{array}{l}4,51^{a b} \\
4,59 a \\
4,32^{b} \\
2,97\left({ }^{2}\right)\end{array}$ & $\begin{array}{l}4,84 \\
4,78 \\
4,70\end{array}$ \\
\hline 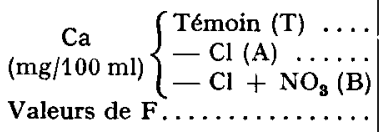 & $\begin{array}{l}22,1 \\
20,7 \\
22,1\end{array}$ & $\begin{array}{l}23,2 \\
22,04 \\
20,8 \\
-\end{array}$ & $\begin{array}{l}25,3 \\
24,6 \\
22,9 \\
1,39\end{array}$ & $\begin{array}{l}22,2 \\
22,3 \\
23,0 \\
-\end{array}$ & $\begin{array}{l}22,7 \\
23,0 \\
22,5\end{array}$ \\
\hline 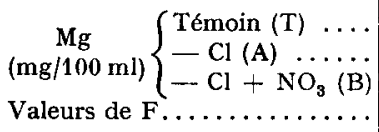 & $\begin{array}{l}3,19 \\
3,02 \\
2,82\end{array}$ & $\begin{array}{l}3,64^{a} \\
2,78^{b} \\
2,56^{b} \\
5,90^{* *}\end{array}$ & $\begin{array}{l}3,44^{a} \\
3,50^{a} \\
3,04^{b} \\
3,66^{*}\end{array}$ & $\begin{array}{l}2,71 \\
2,84 \\
2,89 \\
-\end{array}$ & $\begin{array}{l}3,08 \\
3,00 \\
2,86\end{array}$ \\
\hline $\begin{array}{r}\text { p. Osm. }\left\{\begin{array}{l}\text { Témoin }(\mathrm{T}) \ldots \ldots \\
-\mathrm{Cl}(\mathrm{A}) \ldots \ldots \\
-\mathrm{Cl}+\mathrm{NO}_{3}(\mathrm{~B})\end{array}\right. \\
\text { Valeurs de } \mathrm{F} \ldots \ldots \ldots \ldots \ldots \ldots\end{array}$ & $\begin{array}{l}322 \\
324 \\
324\end{array}$ & $\begin{array}{r}319 \\
317 \\
\mathbf{3 1 6} \\
-\end{array}$ & $\begin{array}{r}317 \\
321 \\
317 \\
-\end{array}$ & $\begin{array}{r}319 \\
317 \\
317 \\
-\end{array}$ & $\begin{array}{l}319 \\
319 \\
318\end{array}$ \\
\hline
\end{tabular}

(1) Les valeurs de $\mathrm{F}$ manquantes sont inférieures d 1.

$a, b:$ les valeurs suivies des mêmes lettres ne sont pas différentes.

(2) $\mathrm{P}<0,1 ; * \mathrm{P}<0,05 ; * * \mathrm{P}<0,01$.

La calcémie n'est pas affectée par les traitements appliqués; à l'opposé, la teneur en magnésium du plasma semble réduite par l'apport de nitrate, tout au moins pendant les 4 premières semaines $(2,56 \mathrm{mg} / \mathrm{I}$ oo $\mathrm{ml}$ en $\mathrm{B}$ contre 3,64 en $\mathrm{T}$ après 
2 semaines). La pression osmotique du plasma des 3 lots diminue de façon identique en début d'expérience, reflétant en cela les variations de la natrémie. Les différents régimes, en tant que tels, n'entraînent aucune modification de pression osmotique sanguine.

Le tableau 3 regroupe les valeurs moyennes des paramètres sanguins pour chacune des deux souches de poules utilisées. En ce qui concerne l'équilibre acidobasique ( $\mathrm{pH}, p \mathrm{CO}_{\mathrm{z}}$ et $\mathrm{HCO}_{\mathrm{s}}{ }^{-}$) on constate que, en moyenne, les poules de type White Leghorn ont une tendance à l'alcalose métabolique par rapport au croisement Rhode $\times$ Wyandotte. Parmi les différences remarquables, on peut aussi souligner une supériorité de la calcémie chez les Leghorn. Ces résultats confirment ceux déjà rapportés (MONGIN et SAUVEUR, I970).

\section{TABLEAU 3}

Effet de la souche de la poule sur l'équilibre acido-basique du sang et la teneur en électrolytes du plasma

(moyenne calculées sur 60 valeurs regroupant tous les traitements)

\begin{tabular}{|c|c|c|c|}
\hline & $\begin{array}{l}\text { White Leghorn } \\
\qquad(H N)\end{array}$ & $\begin{array}{c}\text { Croisement triple } \\
R I R \times \text { Wyandotte } \\
(\mathrm{M} \mathrm{519)}\end{array}$ & $\begin{array}{c}\text { Valeur de } \\
\text { F }_{\text {souche }}\end{array}$ \\
\hline$\ldots \ldots \ldots \ldots$ & 7,435 & 7,373 & $64,0 * * *$ \\
\hline$p \mathrm{CO}_{2}(\mathrm{~mm} \mathrm{Hg}) \ldots$ & 43,7 & 49,0 & $24,0^{* * * *}$ \\
\hline $\mathrm{HCO}_{3}^{-}($méq $/ 1) \ldots$ & 27,4 & 26,1 & $7,3 * *$ \\
\hline $\mathrm{Cl}^{-}(\mathrm{méq} / 1) \ldots$ & 112,5 & 110,8 & $7,9 * *$ \\
\hline $\mathrm{Na}^{+}(\mathrm{méq} / \mathrm{l}) \ldots$ & 149,0 & 149,9 & $3,9 *$ \\
\hline $\mathrm{K}^{+}(\mathrm{méq} / \mathrm{l}) \ldots$ & 4,47 & 4,75 & $26,8 * * *$ \\
\hline $\mathrm{Ca}(\mathrm{mg} / 100 \mathrm{ml})$ & 23,56 & 21,70 & $10,5 * * *$ \\
\hline $\mathrm{Mg}(\mathrm{mg} / 100 \mathrm{ml})$ & 3,10 & 2,95 & $4,6^{*}$ \\
\hline posm (mOsm) $\ldots$. & 318,6 & 319,1 & 0,3 \\
\hline
\end{tabular}

*, **, cf. tableau 2 .

$* * * P<0,001$.

\section{B. - Au niveau de l'œuf}

L'intensité de ponte des troupeaux (nombre d'œufs pondus par jour pour Ioo poules présentes) n'est pas modifiée de façon appréciable par les traitements appliqués : chez les deux souches de poules, elle évolue de $80 \mathrm{p}$. Ioo en début d'expérience à $70 \mathrm{p}$. Ioo après Io semaines, quels que soient les régimes.

Compte tenu des différences entre souches qui se sont manifestées au niveau de la qualité de l'œuf, les résultats qui suivent sont présentés séparément pour chacune des souches. Ces résultats sont exprimés sous forme de variations entre la période expérimentale et la période de pré-expérience : pour ce faire nous avons calculé pour chaque ouf la différence entre la valeur d'un paramètre à l'instant $t$ et la moyenne de la poule pour ce même paramètre à l'instant o (calculée sur les six œufs collectés pendant la période de référence). Les figures 2, 3 et 4 sont composées 
des moyennes par traitement de ces écarts. Cette notation a pour avantage essentiel d'éliminer les différences initiales entre animaux. Le tableau 4 donne par ailleurs les résultats des analyses de variance effectuées.

\section{Poids de l'ouf.}

La figure 2 est relative aux variations du poids de l'œuf. L'effet des régimes y est très hautement significatif $(P<0,00 I)$ de même que l'interaction régime $\times$ souche (tab1. 4). Chez les White Leghorn, la seule réduction de teneur en chlore de l'aliment

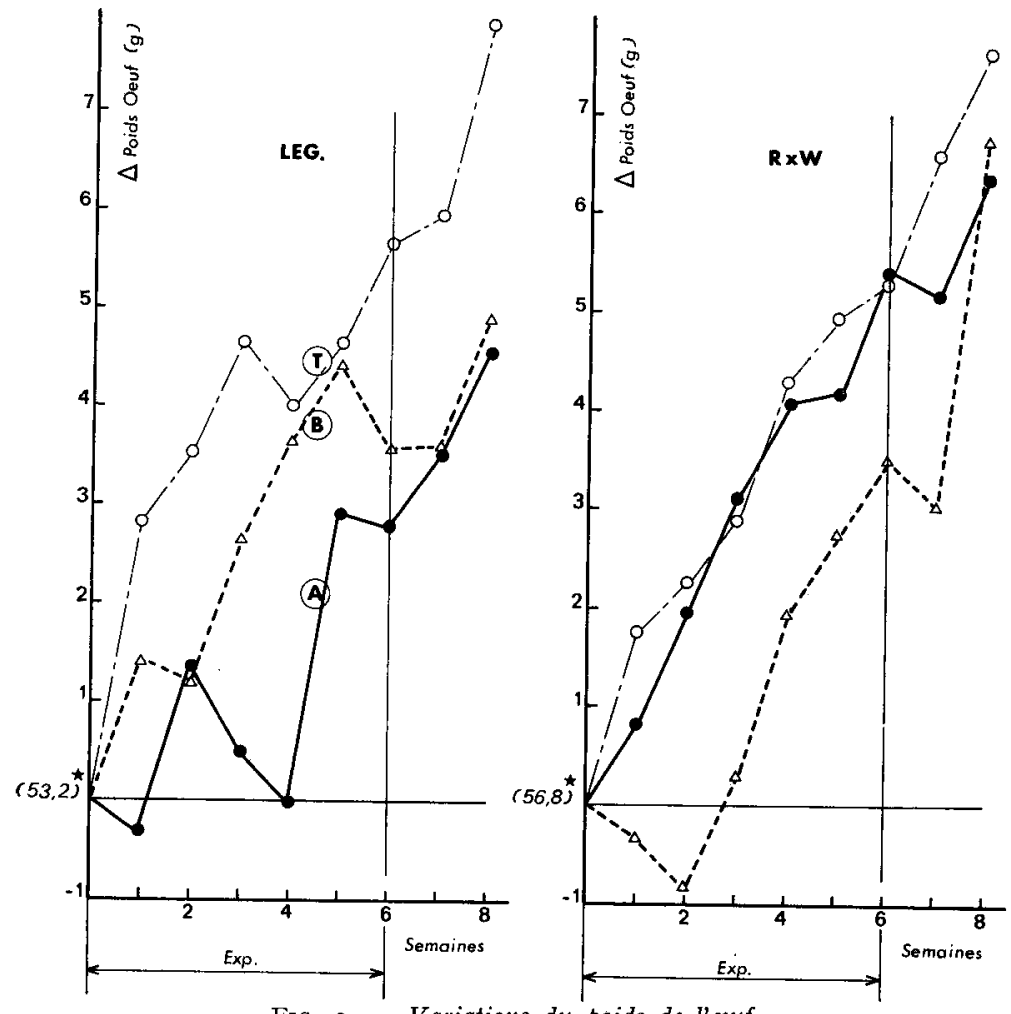

FIG. 2. - Variations du poids de l'œuf

sous l'action de différentes teneurs en chlore et en nitrate de l'aliment

o- - - o lot $\mathrm{T}: 0,24$ p. Ioo $\mathrm{Cl}$

- lot A. : o, I2 p. I0o Cl

$\Delta \ldots . . \Delta$ lot B. : o,I2 p. I0o $\mathrm{Cl}+\mathrm{r}, 5$ p. IOO $\mathrm{NO}_{3}$

Leg. : White Leghorn

$R \times W:$ Rhode Island Red $\times$ Wyandotte

( ) : valeur moyenne de départ

(lot A) arrête la progression normale du poids de l'œuf pendant 4 semaines; à la fin de cette période, le retard par rapport au lot témoin est de $4 \mathrm{~g}$ environ par œuf. La progression du poids de l'œuf reprend ensuite mais l'écart entre les deux lots reste sensiblement le même ; deux semaines après le retour du lot $\mathrm{A}$ au régime témoin (taux chloré élevé), le retard du poids de l'œuf n'est pas rattrapé. L'adjonction de I,5 p. roo de nitrate (lot $B$ ) donne des résultats intermédiaires. 
Les variations de poids de l'œuf induites par les régimes sont différentes chez les poules $R I R \times W$ yandotte. On y observe en effet que la diminution du taux de chlore (lot A) n'affecte en rien la croissance normale du poids de l'œuf. A l'opposé, 1 'adjonction de nitrate (lot $B$ ) entraîne tout d'abord une décroissance absolue de ce poids ( $-\mathrm{I} g$ environ en deux semaines) ; l'augmentation reprend ensuite mais le décalage par rapport au lot témoin subsiste tant que l'aliment contient du nitrate. Après cessation de cet apport le déficit est comblé en deux semaines.

TABLEAU 4

Analyse de variance des modifications des caractéristiques de l'œuf au cours de la période expérimentale

Valeurs de $F$

\begin{tabular}{|c|c|c|c|}
\hline \multirow{2}{*}{ Effet mesuré } & \multicolumn{3}{|c|}{ Critère } \\
\hline & $\Delta$ (Poids de l'cuf) & $\Delta$ (Index de coquille) & $\Delta$ (Unités Haugh) \\
\hline $\begin{array}{l}\text { Régimes } \\
\text { Temps } \\
\text { Souche }\end{array}$ & $\begin{array}{c}38,5 * * * \\
31,9 * * * \\
(1)\end{array}$ & $\begin{array}{c}1,2 \\
1,3 \\
10,1 * *\end{array}$ & $\begin{array}{c}2,09 \\
11,1 * * * \\
1,0\end{array}$ \\
\hline $\begin{array}{c}\text { Régime } \times \text { temps } \\
\text { Temps } \times \text { souche } \\
\text { Régime } \times \text { souche }\left({ }^{2}\right)\end{array}$ & $\begin{array}{l}1,1 \\
2,6^{*} \\
31,3^{* * *}\end{array}$ & $\begin{array}{l}1,5 \\
2,6^{*} \\
38,6^{* * *}\end{array}$ & $\begin{array}{l}\overline{1,2} \\
4,8^{* *}\end{array}$ \\
\hline
\end{tabular}

(1) Les données manquantes de F sont inférieures à 1 .

${ }^{2}{ }^{2}$ Aucune interaction de $2^{e}$ ordre n'est significative.

*, **, ***: cf. tableaux 2 et 3 .

En résumé, le poids de l'œuf apparaît surtout sensible à un éventuel déficit en chlore du régime chez les Leghorn et à un apport de nitrate chez les Rhode $\times$ Wyandotte.

\section{Qualité de la coquille de l'œuf.}

Les variations d'index de coquille des œufs sont représentées à la figure 3 ; là encore, les réponses apparaissent différentes suivant l'origine génétique des animaux comme l'indique l'analyse de variance du tableau 4 (effet souche et interaction régime $\times$ souche hautement significatifs).

Chez les poules White Leghorn, dont l'index moyen originel est élevé $(7,64)$, la restriction de l'apport chloré $(\mathrm{A})$ tend à réduire cet index. Le résultat obtenu avec un apport de nitrate est intermédiaire, comme nous l'avons déjà observé pour le poids de l'œuf.

Les variations d'index de coquille sont beaucoup plus nettes et entièrement différentes chez les poules $R I R \times$ Wyandotte dont l'index moyen de départ est relativement bas $(6,93)$. Dans ce cas la restriction de l'apport chloré (lot $A)$ entraîne $\approx$ ne augmentation spectaculaire de la qualité de la coquille $(+0,6$ point d'index) 
qui permet à ces animaux de rattraper presque totalement l'écart génétique par rapport aux Leghorn. Comme chez ces dernières, 1'apport de nitrate donne encore un résultat intermédiaire en réduisant l'effet bénéfique de la restriction chlorée.

En conclusion, une diminution du taux de chlore de l'aliment apparaît défavorable à la qualité de la coquille chez les Leghorn mais favorable chez les $R I R$ $\times$ Wyandotte. En aucun cas l'adjonction de nitrate ne semble devoir être retenue.
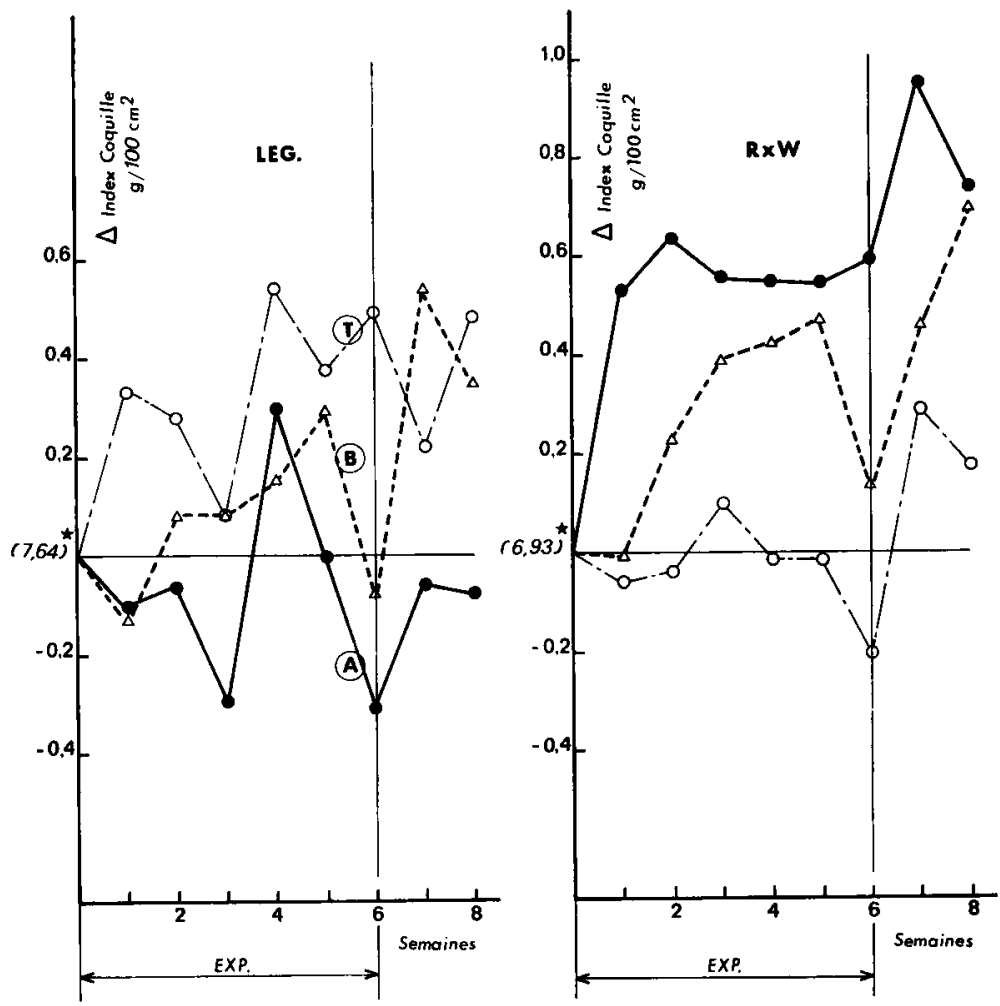

FIG. 3. - Variations de l'index de coquille sous l'action de differentes teneurs en chlore et en nitrate de l'aliment

$$
\begin{aligned}
& \text {-o lot } \mathrm{T} .: \text { o,24 p. Ioo } \mathrm{Cl} \\
& \text { Leg. : White Leghorn } \\
& R \times W \text { : Rhode Island } R e d \times \text { Wyandotte } \\
& (\quad) \text { : valeur moyenne de départ }
\end{aligned}
$$

\section{Qualité de l'albumen de l'œuf.}

La figure 4 et la dernière colonne à droite du tableau 4 sont relatives à la qualité de l'albumen exprimée en unités Haugh. La décroissance des valeurs en fonction $\mathrm{du}$ temps est un phénomène général affectant tous les lots d'animaux. Plus intéressant est l'effet d'interaction régime $\times$ souche $(P<0,0 r)$ qui montre que, là encore, les réponses sont variables suivant l'origine génétique des animaux. Bien que les 

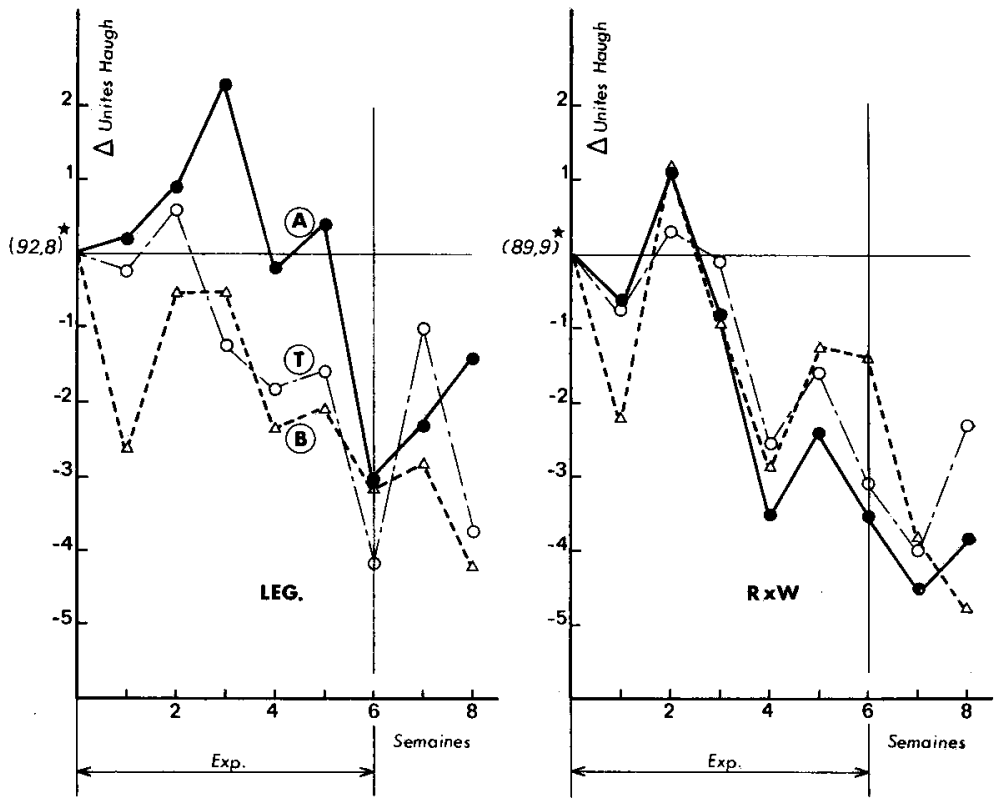

Fig. 4. - Variations de la qualité de l'albumen (Unités Haugh) sous l'action de différentes teneurs en chlore et en nitrate de l'aliment

o- - - $\operatorname{lot} \mathrm{T}: 0,24$ p. roo $\mathrm{Cl}$

- $\longrightarrow$ lot $\mathrm{A}:$ o, I2 p. roo $\mathrm{Cl}$

$\Delta \ldots \Delta$ lot $\mathrm{B}: 0,12$ p. 100 $\mathrm{Cl}+\mathrm{I}, 5$ p. $100 \mathrm{NO}_{3}$

Leg. : White Leghorn

$R \times W:$ Rhode Island Red $\times$ Wyandotte

( ) : valeur moyenne de départ

\section{TABLEAU 5}

Effet de la souche de la poule sur les modifications des caractéristiques de l'ouf au cours de la période expérimentale

(moyennes des 6 semaines de traitement calculées sur 72 valeurs)

\begin{tabular}{|c|c|c|c|}
\hline \multirow[b]{2}{*}{ Critères et Régimes } & \multicolumn{3}{|c|}{ Souche } \\
\hline & $\begin{array}{l}\text { White Leghorn } \\
(H N)\end{array}$ & $\begin{array}{l}\text { Croisement triple } \\
\text { Rhode } \times \text { Wyandotte } \\
(\mathrm{M} \mathrm{519)}\end{array}$ & $\frac{F}{(\text { Régime } \times \text { Race) }}$ \\
\hline $\begin{array}{c}\Delta \text { (poids de l'œuf) } \\
\text { (g) }\end{array}\left\{\begin{array}{l}\text { Témoin (T) } \ldots \ldots \\
-\mathrm{Cl}(\mathrm{A}) \ldots \ldots \\
-\mathrm{Cl}+\mathrm{NO}_{3}(\mathrm{~B})\end{array}\right.$ & $\begin{array}{l}+4,22^{a} \\
+1,21^{b} \\
+\quad 2,79^{c}\end{array}$ & $\begin{array}{l}+3,59^{a} \\
+3,25^{a} \\
+1,27^{b}\end{array}$ & $31,3^{* * *}$ \\
\hline$\underset{\left(\mathrm{g} / 100 \mathrm{~cm}^{2}\right)}{\Delta_{\text {index de coq. }}}\left\{\begin{array}{l}\text { Témoin }(\mathrm{T}) \ldots \ldots \\
-\mathrm{Cl}(\mathrm{A}) \ldots \ldots \\
-\mathrm{Cl}+\mathrm{NO}_{\mathrm{a}}(\mathrm{B})\end{array}\right.$ & $\begin{array}{l}+0,355^{a} \\
-0,078^{b} \\
+0,068^{b}\end{array}$ & $\begin{array}{r}-0,033^{b} \\
+0,566^{c} \\
+0,270^{a}\end{array}$ & $38,6 * * *$ \\
\hline$\Delta$ (unité Haugh) $\left\{\begin{array}{l}\text { Témoin (T) } \ldots \\
-\mathrm{Cl}(\mathrm{A}) \ldots \ldots \\
-\mathrm{Cl}+\mathrm{NO}_{3}(\mathrm{~B})\end{array}\right.$ & $\begin{array}{r}1,41^{a} \\
+\quad 0,09^{b} \\
-1,86^{a}\end{array}$ & $\begin{array}{r}-1,32^{a} \\
-1,62^{a} \\
-1,22^{a}\end{array}$ & $4,8 * *$ \\
\hline
\end{tabular}

**, *** : cf. tableaux 2 et 3. 
différences absolues entre traitements soient faibles, on peut noter en effet que le régime A semble le meilleur pour les Leghorn alors que ceci n'est pas vrai pour les $R I R \times$ Wyandotte. Ainsi les réponses de qualité d'albumen paraissent partiellement opposées à celles enregistrées pour la qualité de la coquille.

\section{Effet de la souche.}

L'ensemble des résultats relatifs à la qualité de l'œuf, est regroupé dans le tableatu 5 qui donne pour les six semaines de traitement, les moyennes par souche des variations enregistrées. On y retrouve clairement que, pour les White Leghorn, l'aliment témoin à $0,24 \mathrm{p}$. Ioo de chlore reste préférable pour le poids de l'œuf et l'index de coquille. Au contraire un taux de 0,12 p. Ioo semble devoir être appliqué aux $R I R \times$ Wyandotte pour optimiser ces deux paramètres. En aucun cas l'apport de nitrate au taux de I,5 p. Ioo n'exerce d'effet intéressant.

\section{C. - Poids corporel}

L'évolution du poids corporel des animaux est donnée par la figure 5 ; chez les deux souches, la réduction de 1'apport de chlore par l'aliment entraîne une perte de poids qui est partiellement compensée par une supplémentation en nitrate. Ce
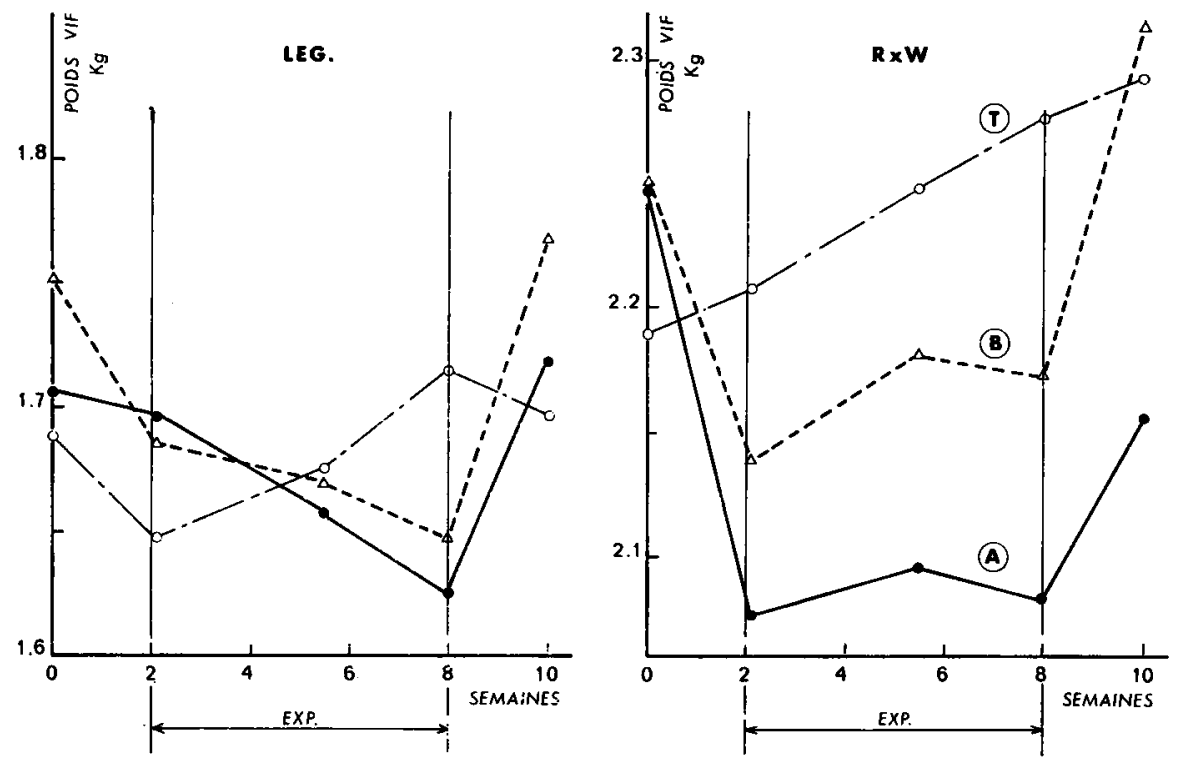

Fig. 5. - Évolution du poids corporel des poules sous l'action de differentes teneurs en chlore et en nitrate de l'aliment

phénomène est beaucoup plus accentué chez les poules $R I R \times$ Wyandotte que chez les White Leghorn. Le poids corporel se rapproche de la valeur normale dès que les animaux consomment à nouveau le régime témoin. 


\section{DISCUSSION}

\section{A. - Chlore, nitrate et alcalose métabolique}

Il a déjà été montré (Mongin, SAUveur et Amin, I972) que la distribution à des poules pondeuses de régimes déficients en chlore provoque l'apparition d'alcaloses métaboliques comme chez les mammifères. La quantité de chlore à incorporer à l'aliment pour éviter ce désordre a pu être fixée aux environs de 0,25 p. Ioo de $\mathrm{NaCl}$ soit 0 ,I2 $\mathrm{p}$. Ioo de $\mathrm{Cl}$ chez des poules du type $R I R \times$ Wyandotte. Les résultats présents sont en bon accord avec les précédents puisque un taux de chlore alimentaire de $0, I 2 \mathrm{p}$. I0o n'induit ici qu'une alcalose légère et transitoire dont il ne reste plus trace après 6 semaines de traitement.

On voit de plus que, en présence d'un apport alimentaire normal en $\mathrm{Na}, \mathrm{K}$ et $\mathrm{Cl}$, l'administration chronique d'une quantité importante de nitrate provoque, chez la Poule, une alcalose métabolique avec hypochlorémie modérées. L’ion nitrate est connu chez les mammifères pour accroître l'élimination urinaire de chlore (HIATT, I940 ; RAPOPORT et WEST, I950). HoL, IDAY (I955) utilisant du $\mathrm{NaNO}_{3}$ en injection péritonéale chez des rats qui reçoivent un régime déficient en $\mathrm{Na}, \mathrm{K}$ et $\mathrm{Cl}$, rapporte de plus un accroissement de la bicarbémie de 10,7 méq/1. Gulyassy et al, (Ig62) qui administrent per os $150 \mathrm{mM} / \mathrm{j}$ de $\mathrm{NaNO}_{3}$ à des chiens, obtiennent des résultats similaires : la bicarbémie s'accroît de ro méq/1 tandis que la chlorémie est diminuée de 20 méq/ 1 après une semaine de traitement.

Dans la présente expérience, la dose administrée par voie orale $\left(25 \mathrm{mM} \mathrm{NO}_{3} /\right.$ jour environ pour des poules dont le poids est compris entre 2 et $2,5 \mathrm{~kg}$ ) est comparable à celle utilisée chez le Chien par Gulyassy et al. (rg62). Dans nos conditions, la bicarbémie augmente au maximum de 5 méq/1 tandis que la chlorémie est réduite de ro méq/1. Bien qu'ayant une amplitude plus faible, cette réponse est bien conforme à celle décrite chez le Rat et le Chien. La différence quantitative de la réaction peut s'expliquer par le fait que nos animaux reçoivent encore une quantité importante de chlore par voie alimentaire, alors que cet apport est totalement supprimé dans les essais précédents. D'ailleurs, un essai précédent effectué avec un régime à $0,24 \mathrm{p}$. Ioo de chlore et $0,9 \mathrm{p}$. Ioo de nitrate n'avait fait apparaître aucune alcalose (résultats non publiés) : la sensibilité de la Poule à $\mathrm{NO}_{3}$ est donc assez faible et ne paraît se manifester que lorsque l'apport chloré est réduit.

D'après les travaux de Gulyassy et al (I962) et surtout ceux plus récents de Wirson et Simmons (I970), il semble que l'alcalose induite par le nitrate soit la conséquence de deux phénomènes : le premier est une contraction des espaces extracellulaires qui se traduit par une salidiurèse importante et une perte de poids corporel. La deuxième origine de 1'accroissement de bicarbémie est probablement le développement d'une acidose intra-cellulaire (WILson et Simmons, r970). Ces effets viendraient se superposer à ceux de la déplétion chlorée dont on sait qu'elle entraîne une augmentation de la réabsorption de bicarbonate par le tubule proximal, au moins à l'état chronique (MELLO-AIres et MALNIC, I972).

I1 est sûr que les résultats présents sont relatifs à un état chronique puisque les premières mesures ont eu lieu deux semaines après introduction du nitrate dans les 
régimes. Par ailleurs, on observe une perte de poids vif mais elle est difficile à interpréter pour les raisons suivantes :

- cette perte est surtout visible chez les poules $R I R \times W$ alors que les deux souches développent une alcalose,

- on ne connaît pas la composition du poids perdu,

- cette perte est plus élevée avec la seule réduction de l'ingéré en chlore qu'après la supplémentation en nitrate génératrice de l'alcalose la plus marquée.

Face à ces données contradictoires, il est difficile de dire quel mécanisme aboutit, chez la Poule, à une augmentation de la bicarbémie après ingestion de nitrate.

La diminution de la kaliémie doit, en revanche, pouvoir s'expliquer par une perte rénale de potassium (Gulyassy et al., I962) ; la baisse de teneur plasmatique en $\mathrm{Mg}$ peut avoir la même origine ou un passage accru de $\mathrm{Mg}$ vers les milieux intra-cellulaires, bien que ce transfert soit supposé faible par WiLson et Srmmons, I970.

\section{B. - Effet du chlore et du nitrate sur la qualité de l'œuf}

\section{Chlore.}

Les travaux antérieurs relatifs à l'action du chlore de l'aliment sur la qualité de la coquille et de l'albumen de l'œuf soulèvent quelques contradictions apparentes ; celles-ci proviennent du fait que, dans la plupart des cas, les auteurs étudient davantage l'effet d'une supplémentation en bicarbonate de sodium que celui du taux de chlore en tant que tel (PEPPER et al., I968; MORRIS, I966 ; Cox et BAI,LOUN, I968). Quand les études portent plus spécifiquement sur l'influence du taux de chlore de l'aliment, elles aboutissent à des conclusions plus claires : ainsi LöRCHER et al. (1964) ont pu montrer qu'un excès de chlore réduit le poids de la coquille. A l'opposé, une réduction de l'apport de chlore sans réduction concomitante de sodium (apporté sous forme de $\mathrm{Na}_{2} \mathrm{CO}_{3}$ ) augmente l'épaisseur de la coquille (Frank et BurGER, I965), le poids de coquille par unité de surface (AMIN, MONGIN et SAUvEUR, I970) et sa résistance à la déformation (BERGER et KUMP, I969; BougON, I97I).

Les résultats présents obtenus avec les poules $R I R \times$ Wyandotte, productrices d'œufs colorés, confirment ces résultats (augmentation de l'index de coquille). A l'opposé, les White Leghorn dont la solidité de coquille initiale est la plus élevée répondent à la réduction de l'apport de chlore par une légère baisse de l'index de coquille. Il apparaît donc là une composante génétique dans la réponse au traitement qu'il est important de retenir.

Nous avons déjà montré que toute situation acido-basique qui crée une diminution stable de la bicarbémie, tend à augmenter la hauteur de l'albumen de l'œuf, exprimée en unités Haugh (SAUVEUR, I970). Inversement cette hauteur diminue si la bicarbémie est élevée (SAUveur, I968; AmIN, MoNGin et SaUveur, I970). Ainsi les variations de hauteur de l'albumen sont, en règle générale, opposées à celle d'épaisseur de la coquille. Ce phénomène se retrouve dans le présent essai; cependant, la faible réduction enregistrée chez les $R I R \times$ Wyandotte n'est pas significative, ce qui laisse toute sa valeur à l'effet largement favorable observé sur la coquille chez cette même souche.

Les résultats relatifs au poids de l'œuf confirment que les deux souches de poules n'ont pas la même sensibilité vis-à-vis de la teneur en chlore du régime. 
Sans que nous puissions l'interpréter, ce résultat conduit à penser que le " besoin " en chlore des Leghorn pour la production de l'œuf serait supérieur à celui des $R I R$ $\times$ Wyandotte.

\section{Nitrate.}

On a vu que l'apport alimentaire de nitrate entraîne, chez les deux souches de poules, une alcalose hypochlorémique. Compte tenu de ce qui précède, on aurait pu s'attendre à ce que, au moins chez la souche à coquille initialement faible $(R I R$ $\times$ Wyandotte), ceci se traduise par une amélioration supplémentaire de l'épaisseur de la coquille. Or ceci n'apparaît pas (fig. 3) : chez les deux souches l'administration de nitrate aboutit à un index de coquille intermédiaire entre celui du lot témoin et celui du lot subissant seulement une réduction du chlore. De plus, ce nitrate réduit la croissance normale du poids de l'œuf (fig. 2). Ce résultat inattendu conduit évidemment à ne pas recommander l'incorporation de nitrate dans les régimes pour poules pondeuses dans les conditions pratiquées ici.

On connaît ainsi plusieurs exemples où apparaît une discordance entre l'équilibre acido-basique général et le résultat attendu au niveau de la formation de la coquille. Ainsi MUELLER (I967) obtient une réduction du dépôt de la coquille par administration de spironolactone sans aucune modification de l'équilibre acidobasique sanguin. AMIN, MONGIN et SAUVEUR (I970), rapportent un accroissement du poids de la coquille par la supplémentation en sulfate d'une ration contenant un niveau normal de chlore, alors que ce traitement n'engendre pas d'alcalose métabolique.

Plusieurs hypothèses peuvent être avancées pour tenter d'interpréter la discordance manifestée dans la présente expérience.

ro Pour garder constants les apports de $\mathrm{Na}$ et $\mathrm{K}$, une grande partie du nitrate a été apportée sous forme de sel de calcium (cf. tabl. I) ; afin de ne pas modifier l'ingéré calcique ceci a obligé à réduire l'apport de $\mathrm{CaCO}_{3}$. Ainsi l'apport de nitrate s'est soldé pour les animaux par une réduction du carbonate ingéré.

$2^{\circ}$ Il est, par ailleurs, possible qu'un apport important d'ion monovalent tel que celui de $\mathrm{NO}_{3}$, doive être accompagné d'une augmentation concomitante de l'ingestion de $\mathrm{Na}$ et $\mathrm{K}$, ce qui n'a pu être effectué ici compte tenu du protocole.

$3^{\circ}$ Enfin Wilson et Simmons (r970) ont montré que, chez le Chien, l'alcalose extracellulaire induite par le nitrate s'accompagne d'une acidose intra-cellulaire avec augmentation de la concentration du sodium à ce niveau. Si une telle acidose intracellulaire est présente au niveau de la muqueuse utérine de la Poule, elle pourrait expliquer localement la réduction de dépôt de carbonate de calcium sur la coquille puisqu'on sait que cette précipitation est vraisemblablement accompagnée d'une réabsorption de $\mathrm{H}^{+}$et de $\mathrm{Na}^{+}$vers les liquides extra-cellulaires (MONGIN, I972).

\section{CONCLUSION}

Il reste vrai qu'une stabilisation chronique de la bicarbémie à un niveau plus élevé que la normale est une condition nécessaire à un accroissement de dépôt de carbonate de calcium sur la coquille de l'œuf : mais cette condition n'est pas toujours 
suffisante comme l'indiquent les résultats obtenus ici après incorporation de nitrate dans le régime des poules, sans modification d'apport des cations. Il apparâtt en outre que le génotype de l'animal semble conditionner le besoin en chlore lié à la production de l'œuf.

Reçu pour publication en avril 1973.

\title{
SUMMARY
}

\author{
NITRATE-INDUCED ALKALOSIS IN THE LAYING HEN
}

It is known that nitrate ion induces a metabolic alkalosis in mammals. The purpose of this experiment was to study whether this ion had the same effect in laying hens and modified the characters of the egg. Three diets containing $0.24 \mathrm{p}$. I00 of chloride (control diet $=\mathrm{T}$ ), 0.12 p. 100 of chloride without nitrate (diet A), and o.I 2 p. Ioo of chloride + I.5 p. roo of nitrate $(\operatorname{diet} B)$, respectively, were compared. The levels of $\mathrm{Ca}, \mathrm{Na}, \mathrm{K}$ and total $\mathrm{P}$ were the same in every diet. During a two-week period, all birds were fed with the control diet; then they were divided in three groups, each of which received during six weeks one of the three diets $T, A$ or B. Thereafter, all birds returned to control diet (T) for two weeks. In each group there were 5 White Leghorn (HN) and 5 Rhode Island Red $\times$ Wyandotte (Selaf M 5I9) hens.

Regardless the strain, nitrate ion added to the low-chloride diet induced a metabolic alkalosis ( $\mathrm{pH}$ and $\mathrm{HCO}_{3}^{-}$level of the blood increased) with hypochloremia (fig. I). During the recovery period, bicarbemia and plasma chloride content returned to normal values. During the alkalosis plasma $\mathrm{K}$ and $\mathrm{Mg}$ contents decreased significantly (tabl. 2).

In White Leghorn hens fed with the low-chloride diet (A), egg weight (fig. 2) and eggshell index (fig. 3) decreased while albumen height expressed as Haugh units slightly increased (fig. 4). Nitrate ion added to the diet (B) reduced the bad effects of the chloride restriction upon egg and shell weights.

The results recorded in brown eggs laid by $\mathrm{M}_{5} \mathrm{I} g$ hens were quite different. In this strain, egg weight and albumen quality were not modified by the chloride restriction (fig. 2) while eggshell quality was increased (fig. 3). In hens receiving nitrate ion in the diet (B), egg weight decreased and the benefit effect of the chloride restriction upon eggshell was reduced.

These results are compared with those obtained in studies of acid-base balance in mammals; relations between acid-base balance and egg formation in birds are also discussed. In practice it is advised to take into account the genotype of the hens before modifying the anion level of the diet.

\section{RÉFÉRENCES BIBLIOGRAPHIQUES}

Amin M., Mongin P., Sauveur B., I97o. Action de différentes doses de chlorure et de sulfate de sodium sur la production de la poule pondeuse et la qualité de l'œuf. Ann. Zootech., 19, 367-383.

Berger R., Kump P., ig69. Der Einfluss verschiedener Futterzusätze auf die Schalenqualität (ermittelt durch Elastizitätsmessungen) des Eies. Arch. Gefiugelk., 33, 444-453.

Bougon M., r97x. Influence du bicarbonate de sodium et du lactate de calcium sur les performances des pondeuses et la qualité des coquilles. Bull. Inform. Sin. exper. A vicult. Ploufragan., 11, 8z-93.

Cox A. C., Balloun S. L., I968. Lack of effect of sodium bicarbonate on shell characteristics. Poult. Sci., 47, I370-1371.

Franck F. R., Burger R. E., r965. The effect of carbon dioxide inhalation and sodium bicarbonate ingestion on egg shell deposition. Poult. Sci., 44, I604-I605.

Gulyassy P. F., Van Ypersele de Strihou C., Schwartz W. B., I962. On the mechanism of nitrateinduced alkalosis. The possible role of selective chloride depletion in acid-base regulation. $\mathrm{J}$. Clin. Invest., 41, I850-I 862 .

Haugh R. E., r937. The Haugh unit for measuring egg quality. U. S. Egg Poult. Mag., 43, $552-555$.

Helbacka N. V., Casterline J. L., Smith C. J., Shaffner C. S., ig64. Investigation of plasma carbonic acid $\mathrm{pK}$ ' of the chicken. Poult. Sci, 41, I850-1862. 
Hiats E. P., 1940. Extreme hypochloremia in dogs induced by nitrate administration. Amer. $J$. Physiol., 129, 597.

Holliday M. A., 1955. Acute metabolic alkalosis : its effect on potassium and acid excretion. J. Clin. invest., 34, 428-433.

Mello Aires M. (de), Malnic G., I972. Micropuncture study of acidification during hypochloremiv alkalosis in the rat. Pflügers Arch., 331, 13-24.

Mongin P., r965. Index de soliditié de coquille de l'œuf. Ses significations. Sa précisior. Ann. Zootet:h., $14,319 \cdot 325$.

Mongin P., 1968. Role of acid-base balance in the physiology of egg shell formation. World's Poull. Sci.J., 24, 200-230.

Mongin P., I972. Communication personnelle.

Mongin P., Lacassagne L., I966. Équilibre acido-basique du sang et formation de la coquille de l'œuf. Ann. Biol. anim. Bioch. Biophys., 6, 93-100.

Mongin P., Sauveur B., r97o, Équilibre acido-basique du sang et composition ionique du plasma chez la poule en hypercapnie chronique. Ann. Biol. anim. Bioch. Biophys., 10, $n^{\circ}$ hors-série 2, I 4 I-I 50.

Mongin P., Sauveur B., Amin M., r972. Alcaloses hypochlorémiques chez la poule pondeuse. Ann. Biol. anim. Bioch. Biophys., 12, 57-67.

Morris, 1966. in Hunt J. R., I970. Fate of ingested sodium bicarbonate in the fowl. Ann. Biol. anim. Bioch. Biophys., 10, no hors-série 2, III-I18.

Mueller W. J., I967. Effects of spironolactone on laying pullets. Poult. Sci., 46, 742-749.

Pepper W. F., Summers J. D., Mc Conachie J. D., rg68. The effect of high levels of calcium, fish products and sodium bicarbonate on egg shell quality. Poult. Sci., 47, 224-229.

RAPOPORT S., West C. D., I950. Ionic antagonism : effect of various anions on chloride excretion during osmotic diuresis in the dog. Amer. J. Physiol., 162, 668.

SAuveur B., I968. Keeping laying hens in $\mathrm{CO}_{2}$ enriched atmosphere. II. The action of $\mathrm{CO}_{2}$ on the albumen quality. Proc. $3^{\text {rd }}$ European Poult. Conf., Jerusalem, 565-566 (Abstr.).

Sauveur B., I97o. Acidoses métaboliques expérimentales chez la poule pondeuse. II. Avtion sur la composition minérale de l'albumen de l'œuf. Ann. Biol. anim. Bioch. Biophys., 10, 81-roo.

Wilson A. F., Simmons D. H., r970, Relationships between potassium, chloride, intracellular and extracellular pH in dogs. Clin. Sci., 39, 731-745. 